\title{
CORRIGENDUM
}

\section{Differential mechanisms of bicalutamide-induced apoptosis in prostate cell lines}

M St John Floyd Jr, SJ Teahan, JM Fitzpatrick and RWG Watson

Prostate Cancer and Prostatic Diseases (2011) 14, 367; doi:10.1038/pcan.2011.44

Correction to: Prostate Cancer and Prostatic Diseases (2009) 12, 25-33; doi:10.1038/pcan.2008.23

In this article, the first author's name was shown incorrectly in the byline and in the correspondence information. The name should have appeared as MS Floyd Jr. 